\title{
Factors Affecting the Limit of Detection For HPLC/Tandem Mass Spectrometry Experiments Based on Gas-phase Ion-Molecule Reactions
}

\section{SUPPORTING INFORMATION}

Leah F. Easterling, Ravikiran Yerabolu, Rashmi Kumar, Kawthar Z. Alzarieni, Hilkka I. Kenttämaa*

Corresponding author:

Hilkka I. Kenttämaa

Address: Department of Chemistry, Purdue University, West Lafayette, IN 47907, USA. Tel.: +1 (765) 494 0882; fax: +1 (765) 4949421.

E-mail: hilkka@purdue.edu

\begin{abstract}
The supporting information includes HPLC/tandem mass spectrometry data for several analytes, details of quantum chemical calculations, a calibration curve for albendazole sulfoxide, aberrant result.
\end{abstract}




\section{Supporting Information}

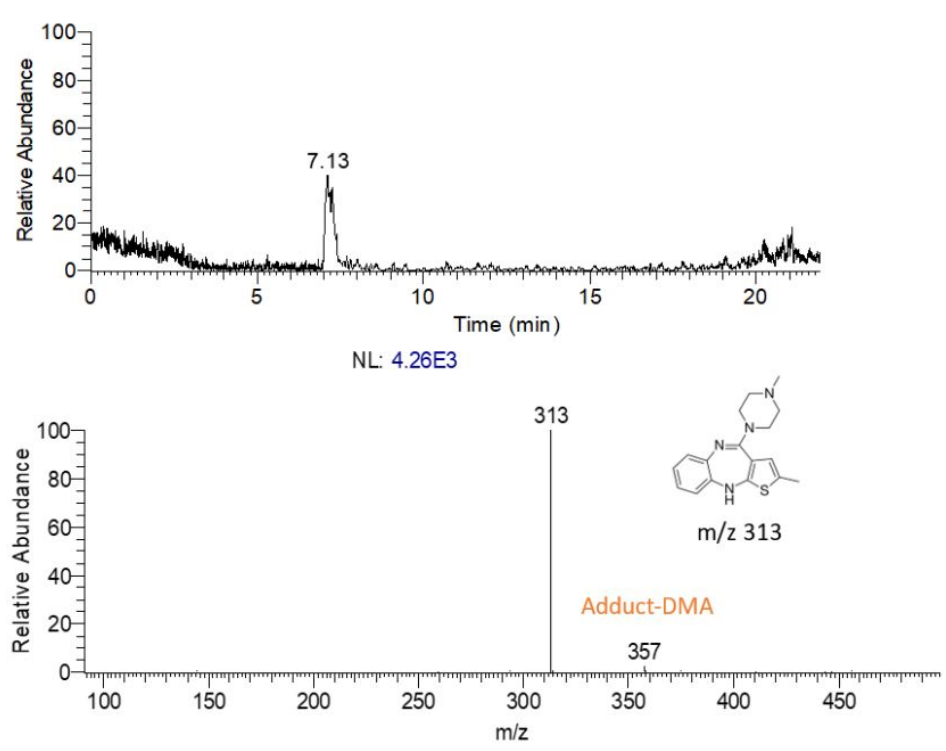

Figure S1. HPLC chromatogram (extracted ion chromatogram for ion of $\mathrm{m} / \mathrm{z} 313$ ) and a MS/MS ion-molecule reaction spectrum measured to determine the limit of detection for Olanzapine. The mass spectrum was obtained at the limit of detection concentration of $100 \mathrm{nM}$. The HPLC chromatogram shows the analyte peak at a retention time of $7.13 \mathrm{~min}$. The MS/MS spectrum obtained using a concentration at the instrument detection limit shows the analyte ion at $\mathrm{m} / \mathrm{z} 313$ and the ion-molecule reaction product of TDMAB and Olanzapine [Adduct-DMA (m/z 357)].
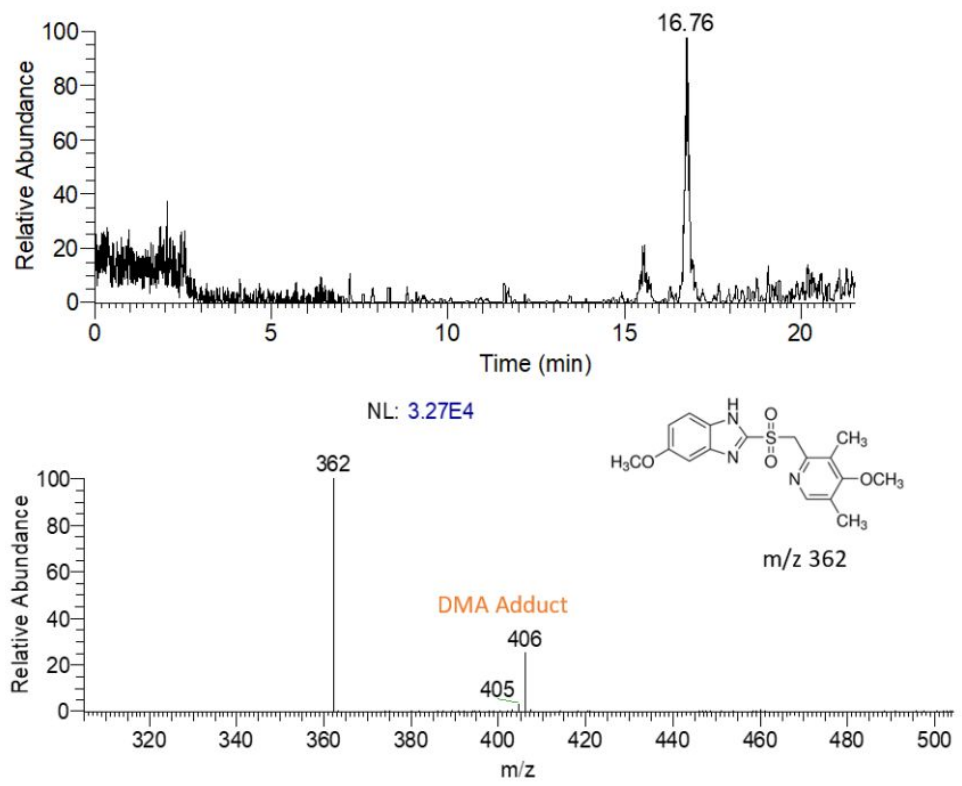

Figure S2. HPLC chromatogram (extracted ion chromatogram for ion of $\mathrm{m} / \mathrm{z} 362$ ) and a tandem mass spectrum measured for Omeprazole Sulfone at the limit of detection: $50 \mathrm{nM}$. The HPLC chromatogram shows the analyte peak at a retention time $16.76 \mathrm{~min}$. and MS/MS spectrum at the 
instrument detection limit shows the analyte ion at m/z 362 and the ion-molecule reaction product of TDMAB and Omeprazole Sulfone [DMA Adduct (m/z 406)].

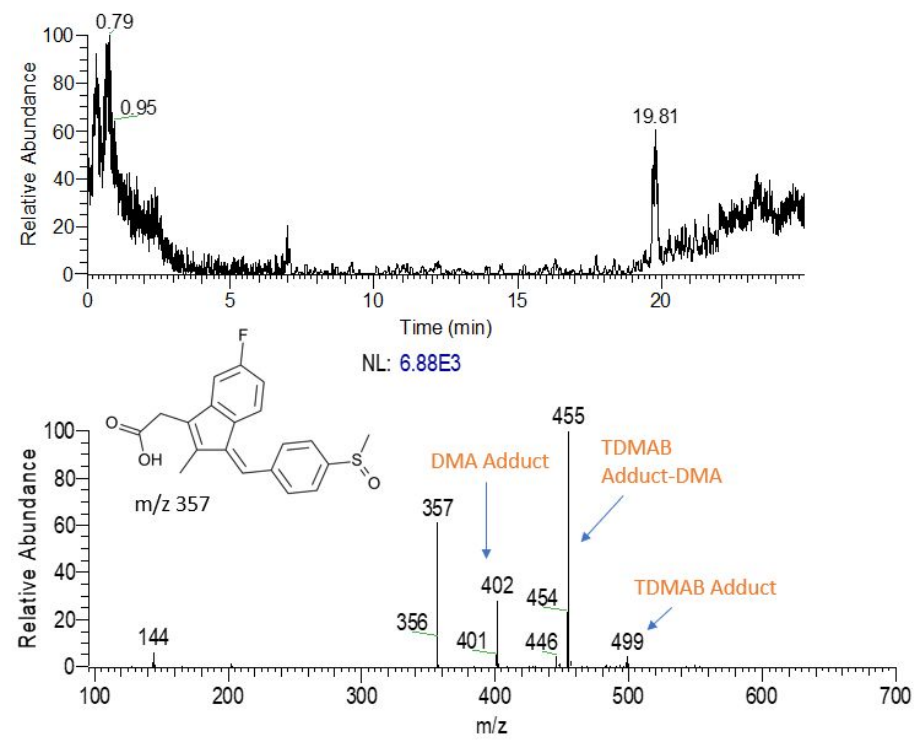

Figure S3. HPLC chromatogram (extracted ion chromatogram for ion of $\mathrm{m} / \mathrm{z} 357$ ) and MS/MS mass spectrum measured for Sulindac at the limit of detection concentration. The MS/MS spectrum was obtained at the limit of detection of $250 \mathrm{nM}$. The HPLC chromatogram shows the analyte peak at retention time $19.81 \mathrm{~min}$. and the mass spectrum measured at the instrument detection limit shows the analyte ion at $\mathrm{m} / \mathrm{z} 357$ and the ion-molecule reaction products of TDMAB and Sulindac [DMA Adduct (m/z 402), TDMAB Adduct-DMA (m/z 455) and TDMAB Adduct (m/z 499)].
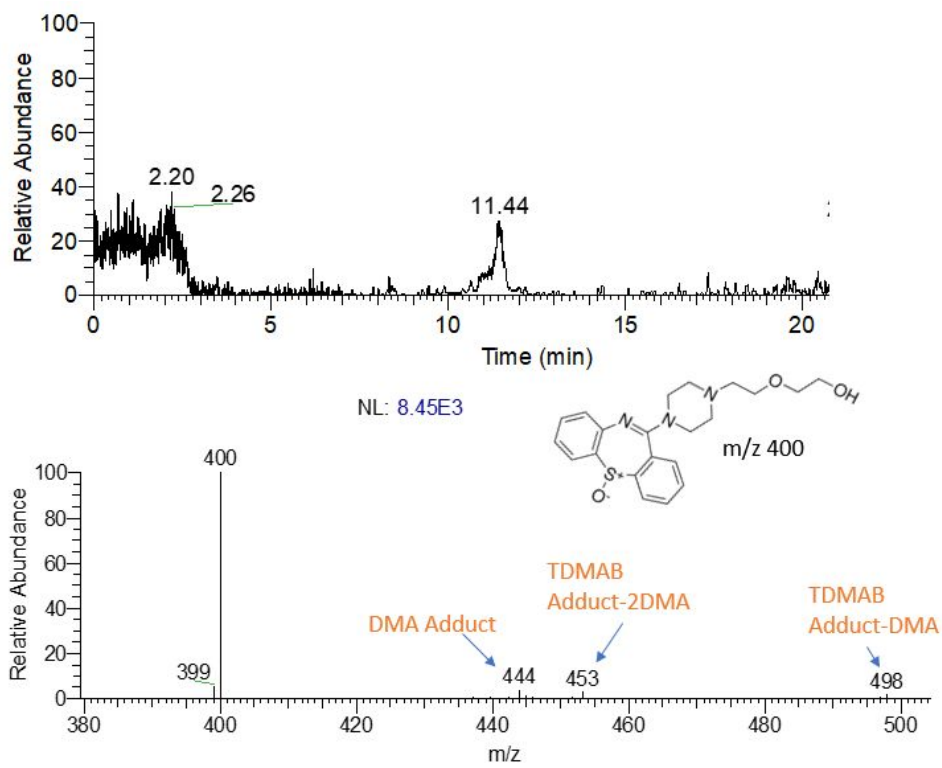

Figure S4. HPLC chromatogram (extracted ion chromatogram for ion of m/z 400) and MS/MS spectrum measure for Quetiapine Sulfoxide at the limit of detection concentration. The MS/MS spectrum was obtained at the limit of detection of $50 \mathrm{nM}$. The HPLC chromatogram shows the analyte peak at a retention time $11.44 \mathrm{~min}$. and the MS/MS spectrum shows the analyte ion at $\mathrm{m} / \mathrm{z}$ 
400 and the ion-molecule reaction products of TDMAB and Quetiapine Sulfoxide [DMA Adduct (m/z 444), TDMAB Adduct-2DMA (m/z 453) and TDMAB Adduct-DMA(m/z 498)].

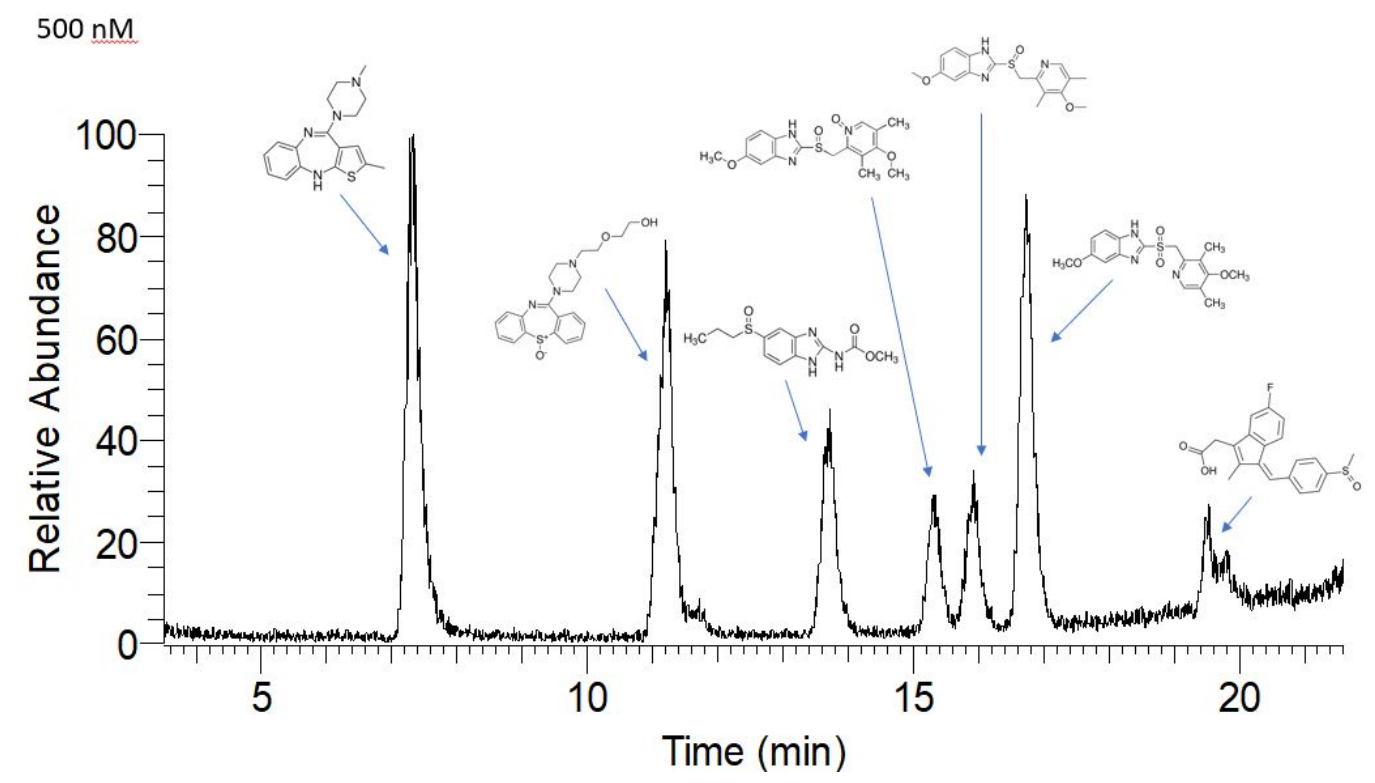

Figure S5. HPLC extracted ion chromatogram of Model Mixture 1 at a concentration of $500 \mathrm{nM}$. The extracted ions were the protonated forms of olanzapine ( $\mathrm{m} / \mathrm{z} 313)$, quetiapine sulfoxide $(\mathrm{m} / \mathrm{z}$ 400), albendazole sulfoxide ( $\mathrm{m} / \mathrm{z} 282)$, omeprazole N-oxide ( $\mathrm{m} / \mathrm{z} 362)$, omeprazole $(\mathrm{m} / \mathrm{z} 346)$, omeprazole sulfone (m/z 362), and sulindac ( $\mathrm{m} / \mathrm{z} 357)$.

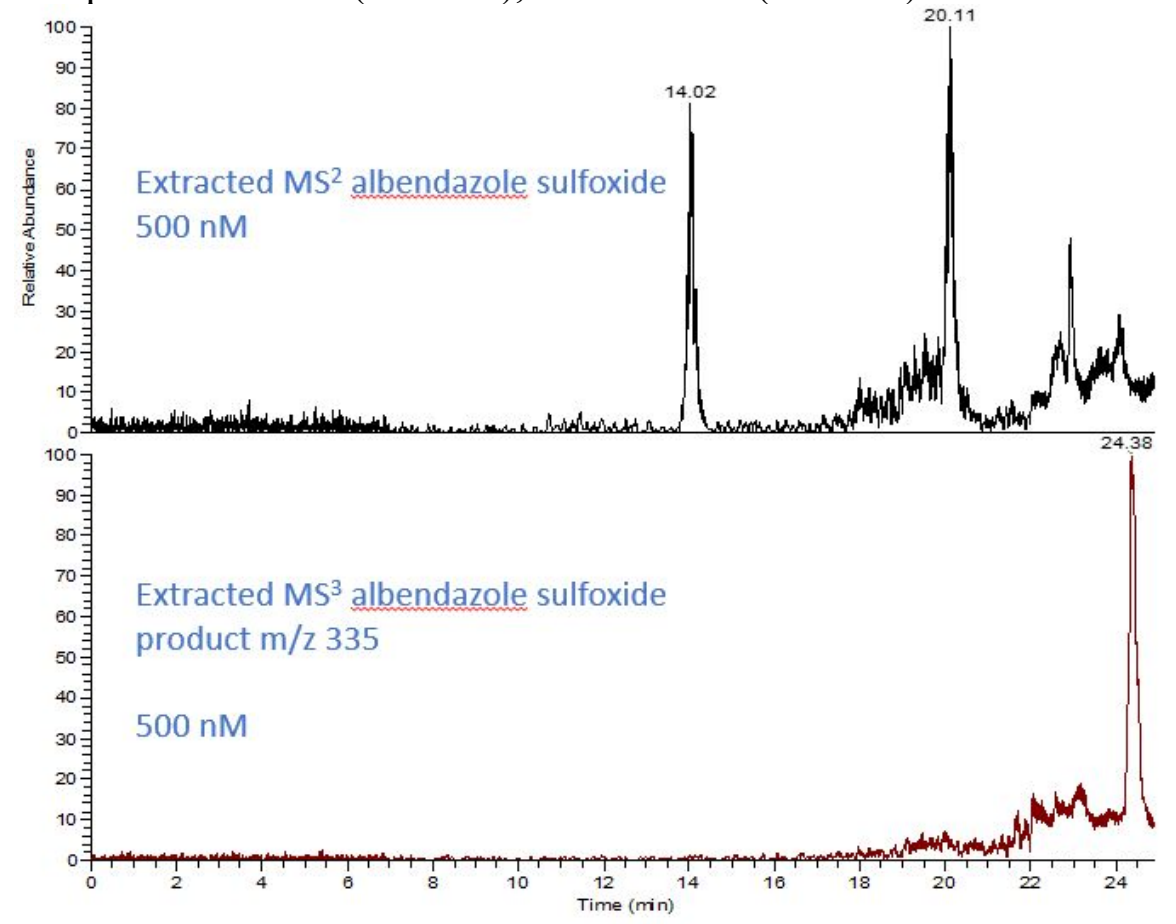




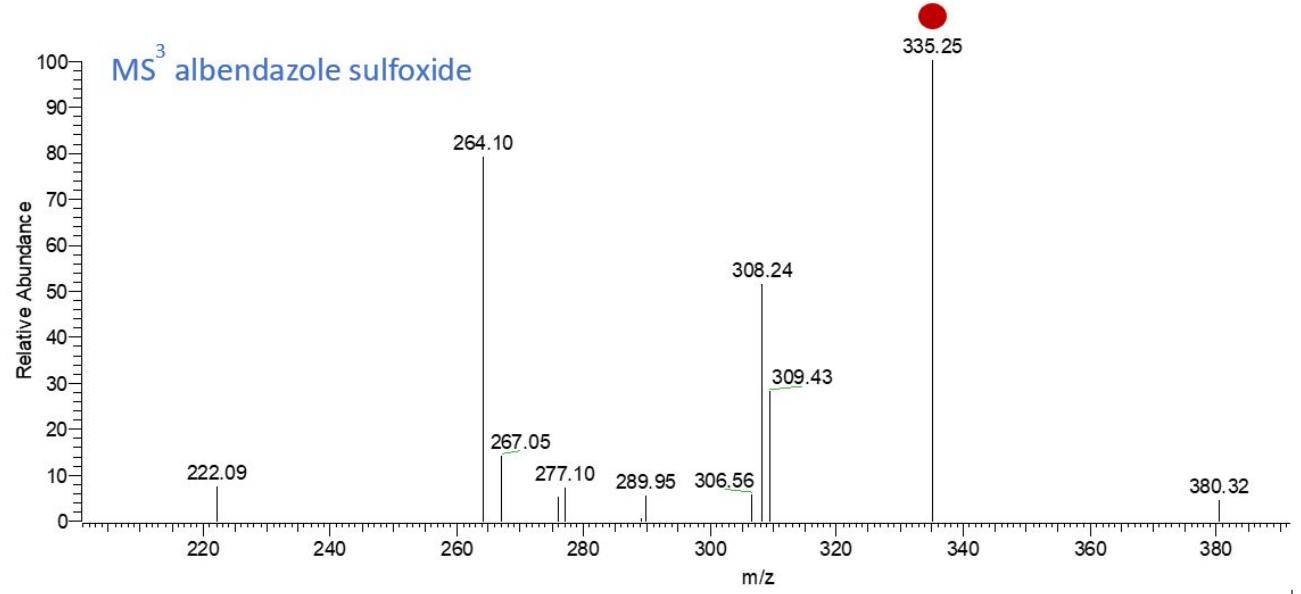

Figure S6. $\mathrm{MS}^{2}$ and $\mathrm{MS}^{3}$ spectra for albendazole sulfoxide at a concentration of $500 \mathrm{nM}$. Top figure shows the extracted ion ( $\mathrm{m} / \mathrm{z} 282)$ HPLC chromatogram, with albendazole sulfoxide eluting at $14.02 \mathrm{~min}$. Bottom figure shows the $\mathrm{MS}^{3}$ spectra obtained after CAD of the most abundant reaction product of ionized albendazole sulfoxide and TDMAB (TDMAB AdductDMA, $\mathrm{m} / \mathrm{z} 380$ ). The spectrum is the extracted ion current of $\mathrm{m} / \mathrm{z} 335$, which is the most consistent and abundant fragment ion of the albendazole sulfoxide TDMAB adduct-DMA ion. The abundance of this fragment ion $(\mathrm{m} / \mathrm{z} 335)$ does not match the elution time of albendazole sulfoxide, suggesting that it is not a real fragment ion of the albendazole sulfoxide TDMAB adduct-DMA product ion.
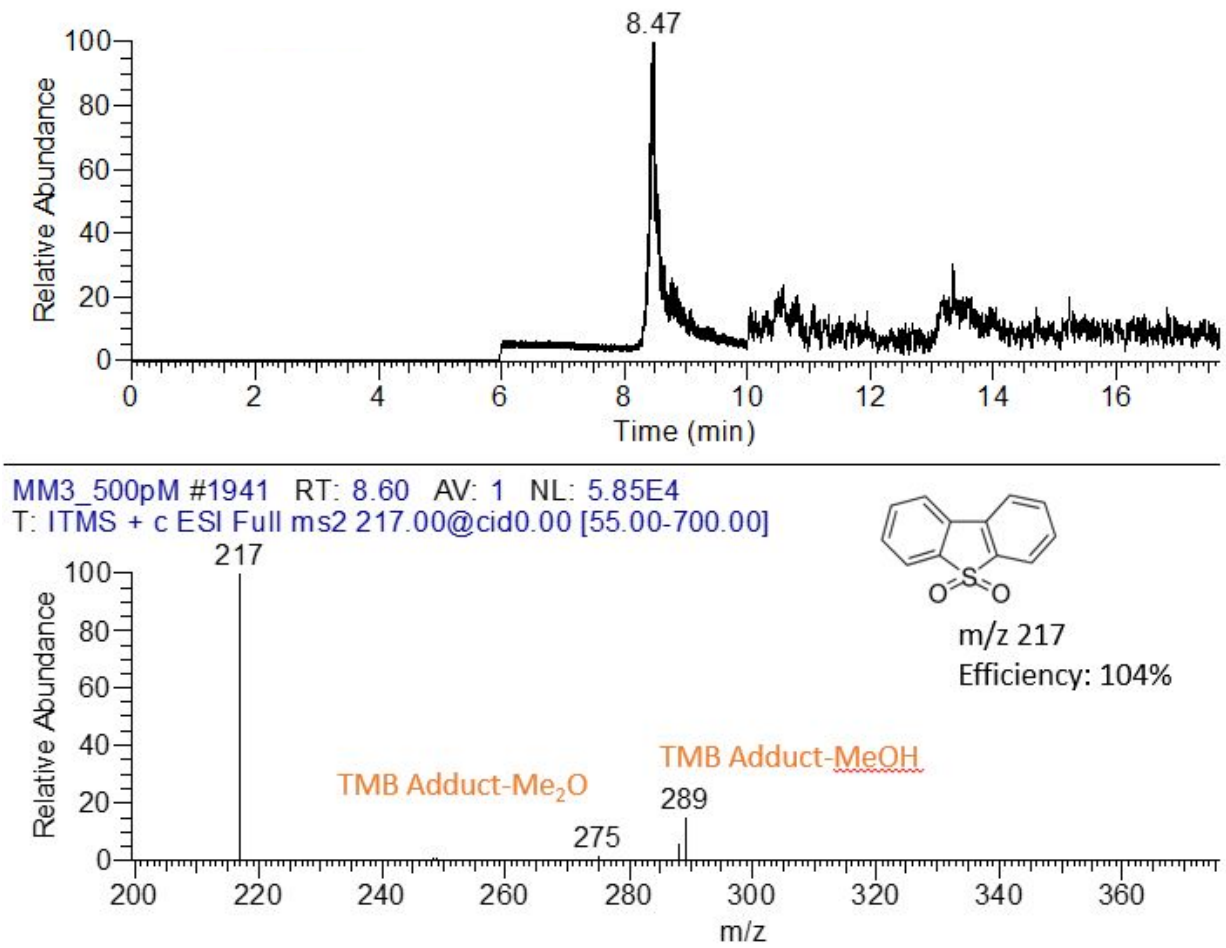
Figure S7. HPLC chromatogram (extracted ion chromatogram for ion of $\mathrm{m} / \mathrm{z} 217$ ) and a tandem mass spectrum measured for protonated dibenzothiophene sulfone at the limit of detection concentration, $50 \mathrm{pM}$. The HPLC chromatogram shows the analyte peak at a retention time 8.47 min. and the tandem mass spectrum shows the analyte ion at $\mathrm{m} / \mathrm{z} 217$ and the ion-molecule reaction products of TMB and dibenzothiophene sulfone [TMB Adduct- $\mathrm{MeOH}(\mathrm{m} / \mathrm{z} 289)$, TMB Adduct$\left.\mathrm{Me}_{2} \mathrm{O}(\mathrm{m} / \mathrm{z} 275)\right]$.
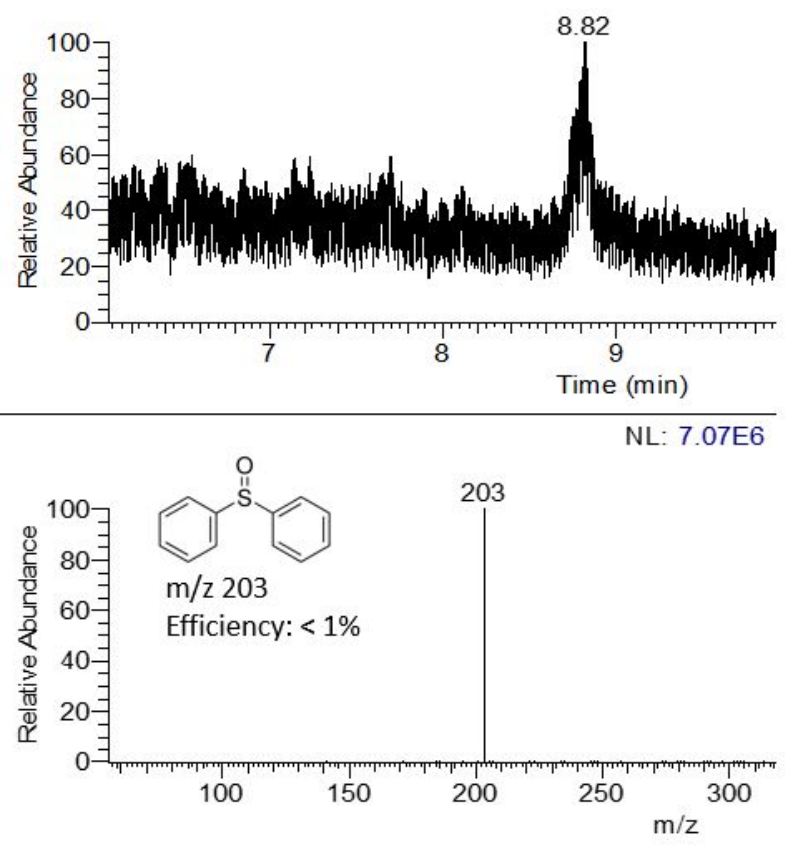

Figure S8. HPLC chromatogram (extracted ion chromatogram for ion of $\mathrm{m} / \mathrm{z} 203$ ) and a tandem mass spectrum measured for protonated diphenylsulfoxide at the limit of detection concentration. The tandem mass spectrum was obtained at the limit of detection of $500 \mathrm{pM}$. The HPLC chromatogram shows the analyte ion peak at a retention time $8.82 \mathrm{~min}$. and the tandem mass spectrum shows the analyte ion at $\mathrm{m} / \mathrm{z} 203$ but no visible reaction products. 
Computational Results 1: Calculated proton affinities (B3LYP/6-31G++ $(\mathrm{d}, \mathrm{p}))$ for all nitrogen, oxygen, and sulfur atoms in optimized omeprazole.

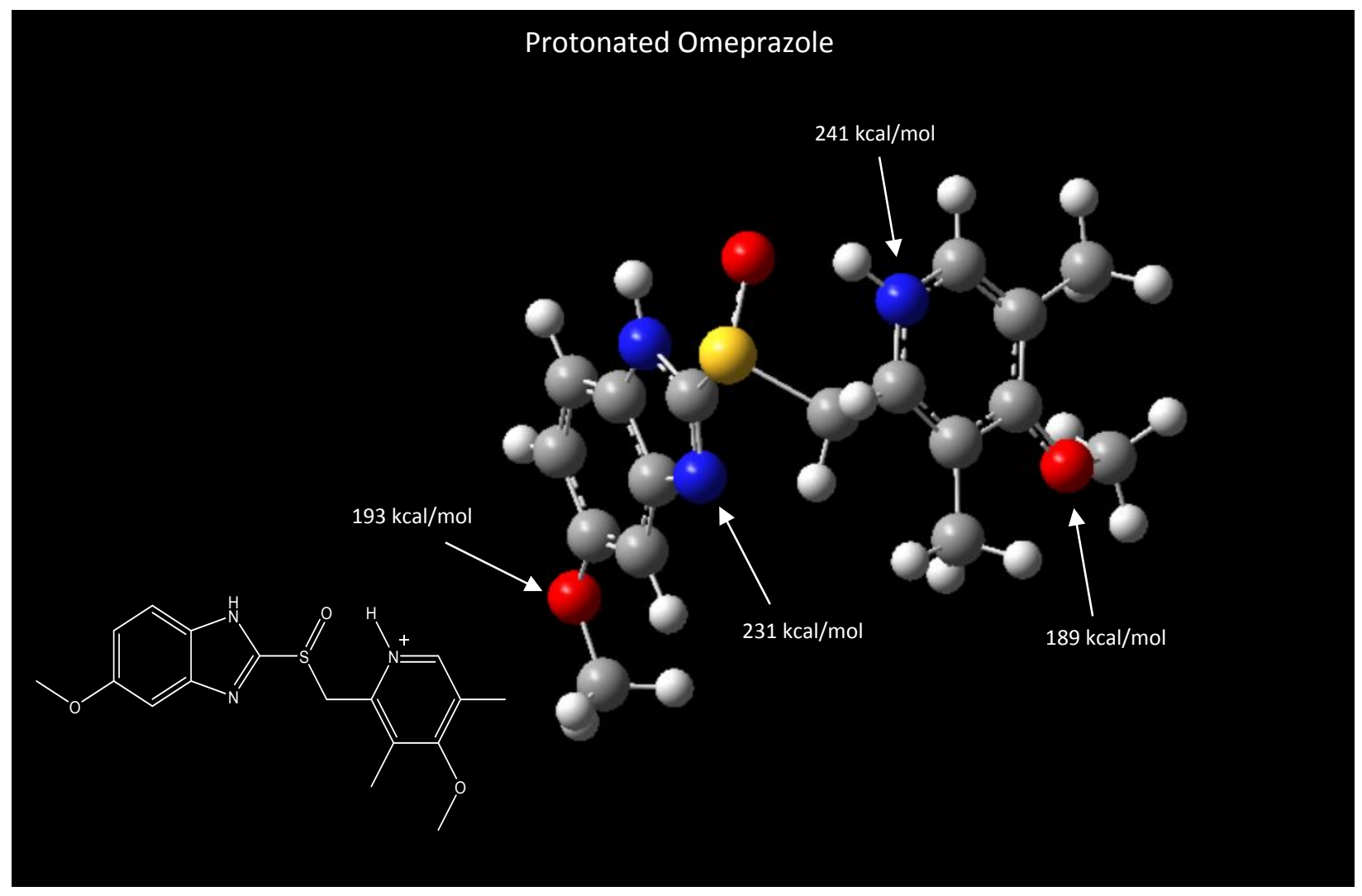

Figure S.: PA values of the most basic sites in omeprazole (shown as protonated) calculated at the B3LYP/6-31G $++(d, p)$ level of theory. Protonation on the sulfoxide group results in the proton moving to the pyridine nitrogen atom.

Neutral Structure

Zero-point energy correction

$=0.339663$

Hartree

Thermal correction to Energy

$=0.363238$

Hartree

Thermal correction to Enthalpy

$=0.364182$

Hartree

Thermal correction to Gibbs Free Energy

$=0.284202$

Hartree

Sum of electronic and zero-point Energies

$=-1447.039097$

Sum of electronic and thermal Energies

$=-1447.015522$

Hartree

Sum of electronic and thermal Enthalpies

$=-1447.014578$

Hartree

Sum of electronic and thermal Free Energies

$=-1447.094668$

Hartree

Hartree 
Protonation on the pyridine nitrogen

$\begin{array}{lll}\text { Zero-point energy correction } & =0.353862 & \text { Hartree } \\ \text { Thermal correction to Energy } & =0.377249 & \text { Hartree } \\ \text { Thermal correction to Enthalpy } & =0.378193 & \text { Hartree } \\ \text { Thermal correction to Gibbs Free Energy } & =0.298886 & \text { Hartree } \\ \text { Sum of electronic and zero-point Energies } & =-1447.419776 & \text { Hartree } \\ \text { Sum of electronic and thermal Energies } & =-1447.396389 & \text { Hartree } \\ \text { Sum of electronic and thermal Enthalpies } & =-1447.395445 & \text { Hartree } \\ \text { Sum of electronic and thermal Free Energies } & =-1447.474752 & \text { Hartree }\end{array}$

\begin{tabular}{|c|c|c|c|}
\hline Omeprazole & \multicolumn{3}{|c|}{ B3LYP/6-31G++(d,p) } \\
\hline \multirow{2}{*}{ Atoms } & \multicolumn{3}{|c|}{ Cartesian coordinates (Angstroms) } \\
\hline & $\mathrm{X}$ & $\mathrm{Y}$ & $\mathrm{Z}$ \\
\hline $\mathrm{C}$ & $-2.2 \mathrm{E}-06$ & $8.47 \mathrm{E}-06$ & $-1.1 \mathrm{E}-05$ \\
\hline $\mathrm{C}$ & $-6.5 \mathrm{E}-06$ & $3.16 \mathrm{E}-07$ & $-4.5 \mathrm{E}-07$ \\
\hline $\mathrm{C}$ & $1.65 \mathrm{E}-05$ & $-2.5 \mathrm{E}-06$ & $-6.9 \mathrm{E}-06$ \\
\hline $\mathrm{C}$ & $-4.7 \mathrm{E}-06$ & 4.59E-06 & $-1.1 \mathrm{E}-06$ \\
\hline $\mathrm{C}$ & $1.09 \mathrm{E}-05$ & $-7.5 \mathrm{E}-06$ & $-3.7 \mathrm{E}-06$ \\
\hline $\mathrm{N}$ & $-7 \mathrm{E}-06$ & $-2.9 \mathrm{E}-06$ & $7.24 \mathrm{E}-06$ \\
\hline $\mathrm{C}$ & $7.75 \mathrm{E}-06$ & $7.31 \mathrm{E}-07$ & $1.62 \mathrm{E}-06$ \\
\hline $\mathrm{S}$ & $2.75 \mathrm{E}-05$ & $-4.7 \mathrm{E}-06$ & $-2.1 \mathrm{E}-07$ \\
\hline $\mathrm{C}$ & $-4.3 \mathrm{E}-05$ & $3.09 \mathrm{E}-06$ & $8.03 \mathrm{E}-06$ \\
\hline $\mathrm{O}$ & $-3.4 \mathrm{E}-06$ & $-6.7 \mathrm{E}-07$ & $2.89 \mathrm{E}-06$ \\
\hline $\mathrm{N}$ & $-9 \mathrm{E}-07$ & $-2.5 \mathrm{E}-06$ & $-2.7 \mathrm{E}-05$ \\
\hline $\mathrm{C}$ & $-1.1 \mathrm{E}-05$ & $-3.9 \mathrm{E}-06$ & $2.8 \mathrm{E}-05$ \\
\hline $\mathrm{C}$ & $4.95 \mathrm{E}-06$ & $1.71 \mathrm{E}-05$ & $-8.1 E-06$ \\
\hline $\mathrm{N}$ & $1.21 \mathrm{E}-05$ & $-5.3 \mathrm{E}-06$ & $-1.1 \mathrm{E}-06$ \\
\hline $\mathrm{C}$ & 7.97E-06 & $-1.6 \mathrm{E}-06$ & $-1.2 \mathrm{E}-05$ \\
\hline $\mathrm{C}$ & $-7.8 \mathrm{E}-06$ & $-9.5 \mathrm{E}-06$ & 7.27E-06 \\
\hline $\mathrm{C}$ & $-4.3 \mathrm{E}-07$ & $5.59 \mathrm{E}-06$ & $-5.1 \mathrm{E}-06$ \\
\hline $\mathrm{C}$ & $4.63 \mathrm{E}-06$ & $-1.6 \mathrm{E}-06$ & 2.4E-07 \\
\hline $\mathrm{O}$ & $-8 \mathrm{E}-08$ & $1.14 \mathrm{E}-06$ & $3.61 \mathrm{E}-07$ \\
\hline $\mathrm{C}$ & $5.27 \mathrm{E}-06$ & $-2 \mathrm{E}-06$ & $-7.1 \mathrm{E}-07$ \\
\hline $\mathrm{C}$ & $2.94 \mathrm{E}-06$ & $-2.1 \mathrm{E}-06$ & 7.03E-06 \\
\hline $\mathrm{O}$ & $-3.3 \mathrm{E}-06$ & $3.54 \mathrm{E}-06$ & $9.99 \mathrm{E}-06$ \\
\hline $\mathrm{C}$ & $-1 \mathrm{E}-05$ & $-4.5 \mathrm{E}-06$ & $1.26 \mathrm{E}-06$ \\
\hline $\mathrm{C}$ & $-1.6 \mathrm{E}-06$ & $5.55 \mathrm{E}-06$ & $1.32 \mathrm{E}-07$ \\
\hline $\mathrm{H}$ & $-2.3 \mathrm{E}-06$ & $1.42 \mathrm{E}-06$ & $-5.5 \mathrm{E}-07$ \\
\hline $\mathrm{H}$ & $-4.3 \mathrm{E}-07$ & $-1.7 \mathrm{E}-06$ & 8.87E-07 \\
\hline $\mathrm{H}$ & $-2.7 \mathrm{E}-06$ & $-1.9 \mathrm{E}-06$ & $-9.8 \mathrm{E}-07$ \\
\hline $\mathrm{H}$ & $7.25 \mathrm{E}-06$ & $3.06 \mathrm{E}-06$ & $1.06 \mathrm{E}-05$ \\
\hline
\end{tabular}




\begin{tabular}{|c|c|c|c|}
\hline $\mathrm{H}$ & $1.01 \mathrm{E}-06$ & $-5.6 \mathrm{E}-07$ & $2.78 \mathrm{E}-06$ \\
\hline $\mathrm{H}$ & $4.42 \mathrm{E}-07$ & $1.2 \mathrm{E}-06$ & $-9.5 \mathrm{E}-07$ \\
\hline $\mathrm{H}$ & $2.74 \mathrm{E}-07$ & $-8.1 \mathrm{E}-07$ & $-8.3 \mathrm{E}-07$ \\
\hline $\mathrm{H}$ & $-2.9 \mathrm{E}-06$ & $-1.8 \mathrm{E}-06$ & $1.11 \mathrm{E}-06$ \\
\hline $\mathrm{H}$ & $-1.6 \mathrm{E}-06$ & $1.14 \mathrm{E}-06$ & $-2.4 \mathrm{E}-06$ \\
\hline $\mathrm{H}$ & $-2.2 \mathrm{E}-06$ & $1.62 \mathrm{E}-06$ & $-1.1 \mathrm{E}-06$ \\
\hline $\mathrm{H}$ & $8.1 \mathrm{E}-08$ & $9.22 \mathrm{E}-07$ & $7.08 \mathrm{E}-07$ \\
\hline $\mathrm{H}$ & $-8.9 \mathrm{E}-07$ & $-7.8 \mathrm{E}-07$ & $-2.6 \mathrm{E}-06$ \\
\hline $\mathrm{H}$ & $9.46 \mathrm{E}-07$ & $1.36 \mathrm{E}-06$ & $-2 \mathrm{E}-06$ \\
\hline $\mathrm{H}$ & $2.5 \mathrm{E}-07$ & $6.38 \mathrm{E}-07$ & $1 \mathrm{E}-07$ \\
\hline $\mathrm{H}$ & $2.63 \mathrm{E}-06$ & $-1.6 \mathrm{E}-06$ & $-1.2 \mathrm{E}-06$ \\
\hline $\mathrm{H}$ & $3.44 \mathrm{E}-06$ & $3.75 \mathrm{E}-06$ & $8.8 \mathrm{E}-08$ \\
\hline $\mathrm{H}$ & $-1.3 \mathrm{E}-07$ & $-1.9 \mathrm{E}-06$ & $-7.7 \mathrm{E}-07$ \\
\hline $\mathrm{H}$ & $-1.1 \mathrm{E}-06$ & $-2.9 \mathrm{E}-06$ & $-9.1 \mathrm{E}-08$ \\
\hline $\mathrm{H}$ & $-1.1 \mathrm{E}-06$ & $2.21 \mathrm{E}-07$ & $1.76 \mathrm{E}-07$ \\
\hline
\end{tabular}

\begin{tabular}{|c|c|c|c|}
\hline \multicolumn{2}{|c|}{ Protonated omeprazole } & \multicolumn{2}{l|}{ B3LYP/6-31G++(d,p) } \\
\hline Atoms & X & Y & Z \\
\hline C & $3.93 \mathrm{E}-06$ & $5.5 \mathrm{E}-06$ & $1.93 \mathrm{E}-06$ \\
\hline C & $-6.4 \mathrm{E}-06$ & $-3 \mathrm{E}-06$ & $7.06 \mathrm{E}-06$ \\
\hline C & $1.87 \mathrm{E}-06$ & $-1.4 \mathrm{E}-06$ & $-8.6 \mathrm{E}-06$ \\
\hline C & $-5.1 \mathrm{E}-07$ & $2.17 \mathrm{E}-06$ & $1.39 \mathrm{E}-06$ \\
\hline C & $2.51 \mathrm{E}-06$ & $5.29 \mathrm{E}-07$ & $-5.1 \mathrm{E}-06$ \\
\hline N & $2.91 \mathrm{E}-06$ & $-4.3 \mathrm{E}-06$ & $2.57 \mathrm{E}-06$ \\
\hline C & $-1.9 \mathrm{E}-06$ & $-8.3 \mathrm{E}-08$ & $3.67 \mathrm{E}-07$ \\
\hline S & $-5.3 \mathrm{E}-07$ & $-1.9 \mathrm{E}-06$ & $1.55 \mathrm{E}-06$ \\
\hline C & $-7.3 \mathrm{E}-07$ & $2.12 \mathrm{E}-06$ & $4.86 \mathrm{E}-07$ \\
\hline O & $1.9 \mathrm{E}-06$ & $-3.9 \mathrm{E}-07$ & $-4.7 \mathrm{E}-06$ \\
\hline N & $6.49 \mathrm{E}-07$ & $-1.7 \mathrm{E}-06$ & $2.27 \mathrm{E}-06$ \\
\hline C & $1.69 \mathrm{E}-06$ & $4.76 \mathrm{E}-06$ & $-2.2 \mathrm{E}-06$ \\
\hline C & $-1.7 \mathrm{E}-06$ & $-3.4 \mathrm{E}-06$ & $-8.2 \mathrm{E}-08$ \\
\hline N & $8.52 \mathrm{E}-07$ & $1.01 \mathrm{E}-06$ & $-1.2 \mathrm{E}-06$ \\
\hline C & $1.54 \mathrm{E}-06$ & $-2.5 \mathrm{E}-06$ & $2.9 \mathrm{E}-06$ \\
\hline C & $-2.9 \mathrm{E}-06$ & $-1.6 \mathrm{E}-06$ & $-3.9 \mathrm{E}-06$ \\
\hline C & $3.97 \mathrm{E}-06$ & $2.37 \mathrm{E}-06$ & $3.3 \mathrm{E}-06$ \\
\hline C & $-1.2 \mathrm{E}-07$ & $-9 \mathrm{E}-07$ & $1.7 \mathrm{E}-06$ \\
\hline O & $-3.9 \mathrm{E}-06$ & $-8.6 \mathrm{E}-07$ & $-1.1 \mathrm{E}-06$ \\
\hline C & $1.66 \mathrm{E}-06$ & $1.28 \mathrm{E}-06$ & $1.63 \mathrm{E}-06$ \\
\hline C & $1.42 \mathrm{E}-06$ & $3.28 \mathrm{E}-06$ & $-1.7 \mathrm{E}-06$ \\
\hline O & $-1.5 \mathrm{E}-06$ & $4.43 \mathrm{E}-06$ & $2.42 \mathrm{E}-06$ \\
\hline C & $-1.2 \mathrm{E}-06$ & $-3.5 \mathrm{E}-06$ & $5.65 \mathrm{E}-07$ \\
\hline & & & \\
\hline
\end{tabular}




\begin{tabular}{|c|c|c|c|}
\hline C & $1.35 \mathrm{E}-06$ & $4.39 \mathrm{E}-07$ & $-1.9 \mathrm{E}-07$ \\
\hline H & $-1.1 \mathrm{E}-07$ & $-2.1 \mathrm{E}-07$ & $-1.5 \mathrm{E}-08$ \\
\hline H & $-6.1 \mathrm{E}-07$ & $-1.8 \mathrm{E}-07$ & $-7.9 \mathrm{E}-07$ \\
\hline H & $1.08 \mathrm{E}-07$ & $8.35 \mathrm{E}-07$ & $4.5 \mathrm{E}-07$ \\
\hline H & $9.66 \mathrm{E}-07$ & $2.67 \mathrm{E}-07$ & $-6.3 \mathrm{E}-07$ \\
\hline H & $1.67 \mathrm{E}-07$ & $5.5 \mathrm{E}-07$ & $-6.6 \mathrm{E}-07$ \\
\hline H & $3.53 \mathrm{E}-07$ & $1.89 \mathrm{E}-07$ & $7.75 \mathrm{E}-07$ \\
\hline H & $-3.6 \mathrm{E}-07$ & $9.8 \mathrm{E}-08$ & $3.72 \mathrm{E}-07$ \\
\hline H & $-6.8 \mathrm{E}-07$ & $-3.7 \mathrm{E}-07$ & $4.16 \mathrm{E}-07$ \\
\hline H & $-7.4 \mathrm{E}-07$ & $-6.6 \mathrm{E}-07$ & $6.42 \mathrm{E}-07$ \\
\hline H & $-8.8 \mathrm{E}-07$ & $-2.4 \mathrm{E}-07$ & $7.05 \mathrm{E}-07$ \\
\hline H & $2.46 \mathrm{E}-07$ & $-1.6 \mathrm{E}-06$ & $1.03 \mathrm{E}-06$ \\
\hline H & $2.3 \mathrm{E}-07$ & $-1.1 \mathrm{E}-06$ & $4.3 \mathrm{E}-08$ \\
\hline H & $-3.3 \mathrm{E}-07$ & $-9.1 \mathrm{E}-07$ & $7.53 \mathrm{E}-07$ \\
\hline H & $-8.9 \mathrm{E}-07$ & $-3.6 \mathrm{E}-07$ & $-7.8 \mathrm{E}-07$ \\
\hline H & $-1.4 \mathrm{E}-06$ & $2.77 \mathrm{E}-07$ & $-1.4 \mathrm{E}-06$ \\
\hline H & $-9.3 \mathrm{E}-07$ & $-1.7 \mathrm{E}-07$ & $-3.3 \mathrm{E}-07$ \\
\hline H & $-9.4 \mathrm{E}-08$ & $-3.7 \mathrm{E}-08$ & $-8.2 \mathrm{E}-07$ \\
\hline H & $8 \mathrm{E}-09$ & $-6 \mathrm{E}-07$ & $-3.2 \mathrm{E}-07$ \\
\hline H & $6.45 \mathrm{E}-07$ & $-5.1 \mathrm{E}-07$ & $-1.7 \mathrm{E}-08$ \\
\hline H & $-5.7 \mathrm{E}-07$ & $2.2 \mathrm{E}-06$ & $-8.3 \mathrm{E}-07$ \\
\hline
\end{tabular}


Computational Results 2: Calculated proton affinities (B3LYP/6-31G++(d,p)) for all nitrogen, oxygen, and sulfur atoms in optimized omeprazole $\mathrm{N}$-oxide.

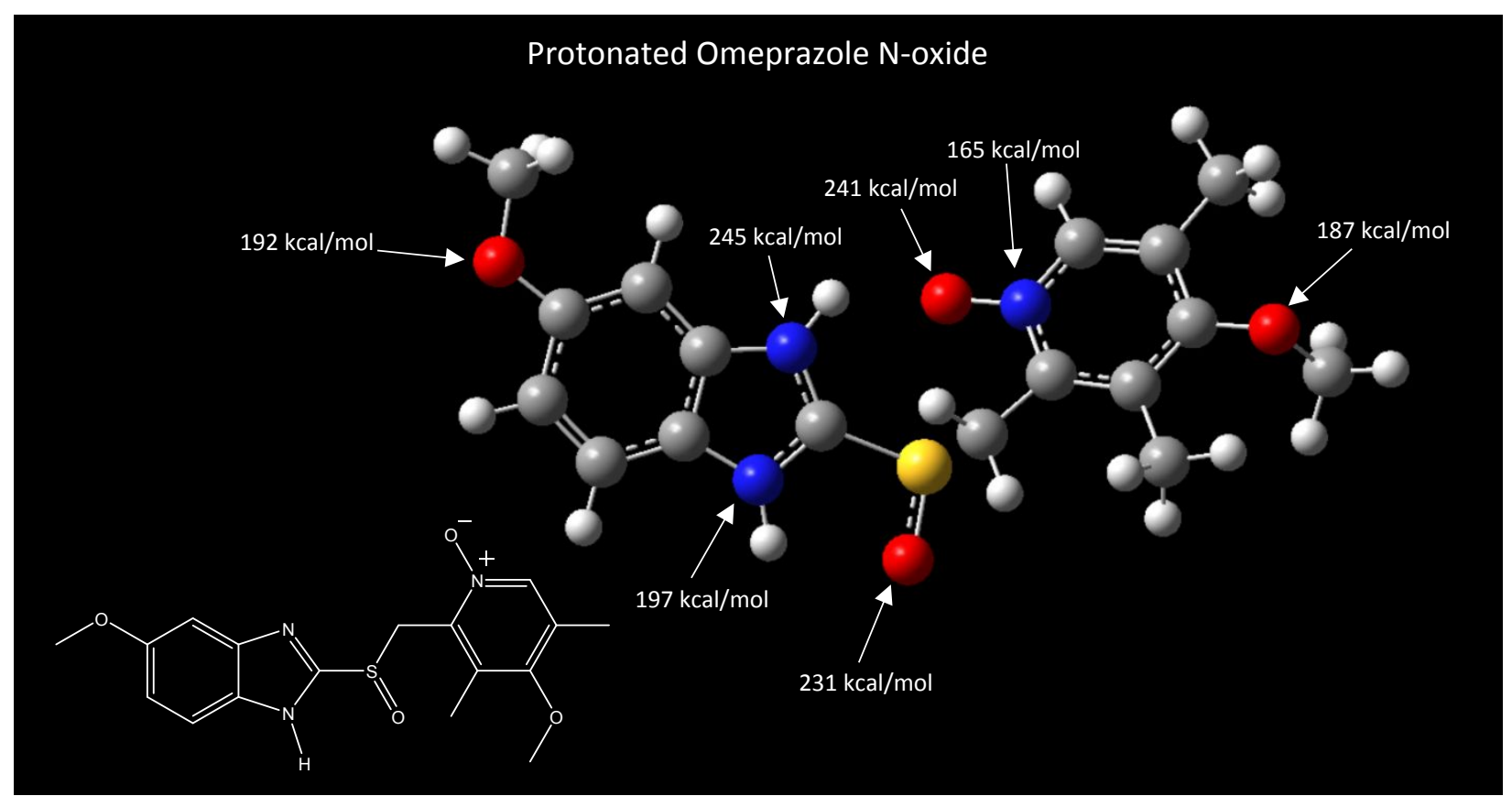

Figure S10. Proton affinities calculated for different protonation sites in omeprazole N-oxide at the B3LYP/6-31G++(d,p) level of theory. The site with the highest proton affinity in omeprazole $\mathrm{N}$-oxide is the nitrogen in the imidazole ring the higher proton affinity of the imine nitrogen can be attributed to intramolecular hydrogen bonding (not shown).

Neutral structure

Zero-point energy correction

Thermal correction to Energy

$=0.343725$

Hartree

Thermal correction to Enthalpy

$=0.368203$

Hartree

Thermal correction to Gibbs Free Energy

$=0.369147$

Hartree

Sum of electronic and zero-point Energies

$=0.287251$

Hartree

Sum of electronic and thermal Energies

$=-1522.201843$

Hartree

Sum of electronic and thermal Enthalpies

$=-1522.177366$

Hartree

Sum of electronic and thermal Free Energies

$=-1522.176421$

Hartree

$=-1522.258317$

Hartree

Protonation on the imidazole imine nitrogen

Zero-point energy correction

$=0.357388$

Hartree

Thermal correction to Energy

$=0.381867$

Hartree

Thermal correction to Enthalpy

$=0.382811$

Hartree

Thermal correction to Gibbs Free Energy

$=0.302326$

Hartree 
Sum of electronic and zero-point Energies

Sum of electronic and thermal Energies

Sum of electronic and thermal Enthalpies

Sum of electronic and thermal Free Energies

$$
\begin{array}{ll}
=-1522.589536 & \text { Hartree } \\
=-1522.565057 & \text { Hartree } \\
=-1522.564112 & \text { Hartree } \\
=-1522.644598 & \text { Hartree }
\end{array}
$$

\begin{tabular}{|c|c|c|c|}
\hline \multirow{2}{*}{ Omeprazole N-oxide } & \multicolumn{2}{c|}{ B3LYP/6-31G++(d,p) } \\
\hline \multirow{2}{*}{ Atoms } & \multicolumn{2}{|c|}{ Cartesian coordinates (Angstroms) } \\
\cline { 2 - 4 } & \multicolumn{2}{|c|}{ X } & \multicolumn{2}{c|}{ y } & Z \\
\hline C & $-9.4 \mathrm{E}-06$ & $1.83 \mathrm{E}-05$ & $3.37 \mathrm{E}-06$ \\
\hline C & $5.28 \mathrm{E}-06$ & $-1.5 \mathrm{E}-05$ & $-3.7 \mathrm{E}-06$ \\
\hline C & $2.13 \mathrm{E}-05$ & $1.34 \mathrm{E}-05$ & $-3.2 \mathrm{E}-06$ \\
\hline C & $-2.3 \mathrm{E}-05$ & $-2.6 \mathrm{E}-05$ & $4.35 \mathrm{E}-06$ \\
\hline C & $-5.1 \mathrm{E}-06$ & $7.31 \mathrm{E}-07$ & $1.04 \mathrm{E}-05$ \\
\hline C & $1.54 \mathrm{E}-05$ & $-5.1 \mathrm{E}-07$ & $-7.5 \mathrm{E}-06$ \\
\hline N & $-2 \mathrm{E}-05$ & $3.68 \mathrm{E}-05$ & $1.03 \mathrm{E}-05$ \\
\hline C & $2.96 \mathrm{E}-05$ & $-8.4 \mathrm{E}-06$ & $-8.4 \mathrm{E}-07$ \\
\hline N & $-1.5 \mathrm{E}-05$ & $-4.5 \mathrm{E}-07$ & $-2 \mathrm{E}-05$ \\
\hline S & $-3.2 \mathrm{E}-06$ & $-9.9 \mathrm{E}-06$ & $-1.2 \mathrm{E}-05$ \\
\hline O & $4.96 \mathrm{E}-06$ & $-9.5 \mathrm{E}-06$ & $-8.3 \mathrm{E}-06$ \\
\hline C & $1.1 \mathrm{E}-05$ & $1.61 \mathrm{E}-05$ & $-8.5 \mathrm{E}-06$ \\
\hline C & $5.4 \mathrm{E}-07$ & $-3.3 \mathrm{E}-05$ & $1.9 \mathrm{E}-05$ \\
\hline C & $7.83 \mathrm{E}-06$ & $1.21 \mathrm{E}-05$ & $-8.5 \mathrm{E}-06$ \\
\hline C & $-2.6 \mathrm{E}-06$ & $-2 \mathrm{E}-06$ & $4.91 \mathrm{E}-07$ \\
\hline C & $-6.9 \mathrm{E}-07$ & $-6.5 \mathrm{E}-08$ & $1.06 \mathrm{E}-06$ \\
\hline C & $2.57 \mathrm{E}-05$ & $-3.7 \mathrm{E}-08$ & $-4 \mathrm{E}-06$ \\
\hline N & $-1.3 \mathrm{E}-05$ & $7.96 \mathrm{E}-06$ & $4.12 \mathrm{E}-07$ \\
\hline C & $-1.2 \mathrm{E}-05$ & $9.19 \mathrm{E}-06$ & $-5.9 \mathrm{E}-06$ \\
\hline O & $-5.5 \mathrm{E}-06$ & $3.2 \mathrm{E}-06$ & $5.52 \mathrm{E}-06$ \\
\hline C & $-7.6 \mathrm{E}-07$ & $5.7 \mathrm{E}-06$ & $4 \mathrm{E}-06$ \\
\hline C & $-8.2 \mathrm{E}-06$ & $-2.8 \mathrm{E}-06$ & $9.75 \mathrm{E}-07$ \\
\hline O & $-8.8 \mathrm{E}-07$ & $2.54 \mathrm{E}-06$ & $-9.7 \mathrm{E}-07$ \\
\hline O & $-2.3 \mathrm{E}-06$ & $-8.4 \mathrm{E}-06$ & $-4.5 \mathrm{E}-07$ \\
\hline C & $-4 \mathrm{E}-06$ & $-7.4 \mathrm{E}-07$ & $1.27 \mathrm{E}-06$ \\
\hline H & $2.01 \mathrm{E}-06$ & $-1.8 \mathrm{E}-06$ & $-7.9 \mathrm{E}-07$ \\
\hline H & $-1.4 \mathrm{E}-06$ & $-2.5 \mathrm{E}-06$ & $-1.7 \mathrm{E}-08$ \\
\hline H & $4.08 \mathrm{E}-07$ & $1.87 \mathrm{E}-07$ & $4.16 \mathrm{E}-07$ \\
\hline H & $-2.8 \mathrm{E}-06$ & $-2.2 \mathrm{E}-06$ & $1.26 \mathrm{E}-05$ \\
\hline H & $-3.2 \mathrm{E}-06$ & $2.58 \mathrm{E}-06$ & $1.91 \mathrm{E}-06$ \\
\hline H & $-2.2 \mathrm{E}-06$ & $-4.6 \mathrm{E}-06$ & $1.07 \mathrm{E}-05$ \\
\hline & $-2.7 \mathrm{E}-06$ & $1.55 \mathrm{E}-06$ & $7.96 \mathrm{E}-07$ \\
\hline & $8.76 \mathrm{E}-06$ & $-6.6 \mathrm{E}-06$ & $1.2 \mathrm{E}-06$ \\
\hline
\end{tabular}




\begin{tabular}{|c|c|c|c|}
\hline $\mathrm{H}$ & $6.72 \mathrm{E}-07$ & $2.62 \mathrm{E}-06$ & $6.11 \mathrm{E}-06$ \\
\hline $\mathrm{H}$ & $3.41 \mathrm{E}-07$ & $-2 \mathrm{E}-06$ & $-2.8 \mathrm{E}-06$ \\
\hline $\mathrm{H}$ & $4.19 \mathrm{E}-06$ & $-3.3 \mathrm{E}-06$ & $2.56 \mathrm{E}-06$ \\
\hline $\mathrm{H}$ & $-4.1 \mathrm{E}-06$ & $-2.7 \mathrm{E}-06$ & $-3.8 \mathrm{E}-06$ \\
\hline $\mathrm{H}$ & $-8.6 \mathrm{E}-07$ & $6.01 \mathrm{E}-06$ & $-1.7 \mathrm{E}-07$ \\
\hline $\mathrm{H}$ & $1.63 \mathrm{E}-06$ & $-1.2 \mathrm{E}-06$ & $5.9 \mathrm{E}-06$ \\
\hline $\mathrm{H}$ & $-7 \mathrm{E}-07$ & $-3.4 \mathrm{E}-06$ & $-5.4 \mathrm{E}-06$ \\
\hline $\mathrm{H}$ & $-4.3 \mathrm{E}-06$ & $-2 \mathrm{E}-07$ & $1.23 \mathrm{E}-06$ \\
\hline $\mathrm{H}$ & $4.57 \mathrm{E}-06$ & $2.47 \mathrm{E}-06$ & $3.74 \mathrm{E}-06$ \\
\hline $\mathrm{H}$ & $2.39 \mathrm{E}-06$ & $3.44 \mathrm{E}-06$ & $-4.9 \mathrm{E}-06$ \\
\hline $\mathrm{H}$ & $1.08 \mathrm{E}-06$ & $1.57 \mathrm{E}-06$ & $-6.3 \mathrm{E}-06$ \\
\hline
\end{tabular}

\begin{tabular}{|c|c|c|c|}
\hline \multirow{2}{*}{$\begin{array}{c}\text { Protonated omeprazole } \\
\text { N-oxide }\end{array}$} & \multicolumn{2}{c|}{ B3LYP/6-31G++(d,p) } \\
\hline \multirow{2}{*}{ Atoms } & \multicolumn{2}{|c|}{ Cartesian coordinates (Angstroms) } \\
\cline { 2 - 4 } & $\mathrm{x}$ & $\mathrm{y}$ & $\mathrm{Z}$ \\
\hline C & $-6.6 \mathrm{E}-06$ & $-2.2 \mathrm{E}-05$ & $1.19 \mathrm{E}-06$ \\
\hline C & $1.35 \mathrm{E}-05$ & $2.3 \mathrm{E}-05$ & $-2.8 \mathrm{E}-06$ \\
\hline C & $1.27 \mathrm{E}-05$ & $-1.3 \mathrm{E}-05$ & $-2.3 \mathrm{E}-06$ \\
\hline C & $-1.7 \mathrm{E}-05$ & $3.86 \mathrm{E}-06$ & $6.58 \mathrm{E}-06$ \\
\hline C & $-8.2 \mathrm{E}-06$ & $-3.3 \mathrm{E}-06$ & $9.79 \mathrm{E}-06$ \\
\hline C & $1.15 \mathrm{E}-05$ & $8.99 \mathrm{E}-06$ & $-1.2 \mathrm{E}-06$ \\
\hline N & $-5.3 \mathrm{E}-06$ & $1.33 \mathrm{E}-05$ & $1.22 \mathrm{E}-06$ \\
\hline C & $6.24 \mathrm{E}-06$ & $-2.2 \mathrm{E}-05$ & $1.16 \mathrm{E}-05$ \\
\hline N & $4.48 \mathrm{E}-06$ & $-4.2 \mathrm{E}-07$ & $-1.5 \mathrm{E}-05$ \\
\hline S & $2.32 \mathrm{E}-06$ & $1.42 \mathrm{E}-05$ & $-2.5 \mathrm{E}-05$ \\
\hline O & $1.07 \mathrm{E}-06$ & $6.82 \mathrm{E}-06$ & $-5.8 \mathrm{E}-06$ \\
\hline C & $2.79 \mathrm{E}-05$ & $-2.7 \mathrm{E}-05$ & $2.06 \mathrm{E}-05$ \\
\hline C & $-2 \mathrm{E}-06$ & $3.3 \mathrm{E}-05$ & $2.57 \mathrm{E}-05$ \\
\hline C & $4.48 \mathrm{E}-06$ & $3.7 \mathrm{E}-06$ & $-4.6 \mathrm{E}-06$ \\
\hline C & $-3.1 \mathrm{E}-06$ & $1.24 \mathrm{E}-06$ & $-3.9 \mathrm{E}-06$ \\
\hline C & $6.94 \mathrm{E}-06$ & $2.53 \mathrm{E}-07$ & $-9.2 \mathrm{E}-07$ \\
\hline C & $-1.6 \mathrm{E}-06$ & $-1 \mathrm{E}-05$ & $-1.3 \mathrm{E}-06$ \\
\hline N & $6.91 \mathrm{E}-06$ & $-5.9 \mathrm{E}-06$ & $-1.9 \mathrm{E}-07$ \\
\hline C & $-4.5 \mathrm{E}-06$ & $-3.9 \mathrm{E}-06$ & $-8.1 \mathrm{E}-06$ \\
\hline O & $-2.4 \mathrm{E}-06$ & $-5.3 \mathrm{E}-07$ & $6.25 \mathrm{E}-06$ \\
\hline C & $-5.2 \mathrm{E}-06$ & $-4.4 \mathrm{E}-06$ & $1.95 \mathrm{E}-06$ \\
\hline C & $-3.8 \mathrm{E}-06$ & $8.04 \mathrm{E}-06$ & $3.19 \mathrm{E}-07$ \\
\hline O & $-6.8 \mathrm{E}-06$ & $-1.7 \mathrm{E}-06$ & $-1.1 \mathrm{E}-05$ \\
\hline O & $-1.4 \mathrm{E}-05$ & $4.63 \mathrm{E}-06$ & $1.65 \mathrm{E}-06$ \\
\hline C & $4.06 \mathrm{E}-06$ & $8.41 \mathrm{E}-07$ & $-1.5 \mathrm{E}-06$ \\
\hline H & $-7.3 \mathrm{E}-07$ & $3.94 \mathrm{E}-06$ & $-6.5 \mathrm{E}-07$ \\
\hline
\end{tabular}




\begin{tabular}{|c|c|c|c|}
\hline $\mathrm{H}$ & $-6.7 \mathrm{E}-07$ & $-1.8 \mathrm{E}-06$ & $7.7 \mathrm{E}-08$ \\
\hline $\mathrm{H}$ & $-2.5 \mathrm{E}-06$ & $5.95 \mathrm{E}-07$ & $7.72 \mathrm{E}-07$ \\
\hline $\mathrm{H}$ & $-1.3 \mathrm{E}-05$ & $-2.5 \mathrm{E}-06$ & $3.49 \mathrm{E}-06$ \\
\hline $\mathrm{H}$ & $-5.5 \mathrm{E}-06$ & $-7.2 \mathrm{E}-06$ & $-4.9 \mathrm{E}-06$ \\
\hline $\mathrm{H}$ & $5.21 \mathrm{E}-06$ & $6.91 \mathrm{E}-06$ & $5.61 \mathrm{E}-06$ \\
\hline $\mathrm{H}$ & $-7.8 \mathrm{E}-07$ & $-9.9 \mathrm{E}-07$ & $-1.5 \mathrm{E}-06$ \\
\hline $\mathrm{H}$ & $6.67 \mathrm{E}-06$ & $-3.9 \mathrm{E}-06$ & $1.03 \mathrm{E}-06$ \\
\hline $\mathrm{H}$ & $-3.7 \mathrm{E}-06$ & $-1.3 \mathrm{E}-06$ & $6.73 \mathrm{E}-06$ \\
\hline $\mathrm{H}$ & $-2.8 \mathrm{E}-06$ & $2.97 \mathrm{E}-06$ & $-3.1 \mathrm{E}-06$ \\
\hline $\mathrm{H}$ & $3.6 \mathrm{E}-06$ & $-2 \mathrm{E}-06$ & $3.73 \mathrm{E}-06$ \\
\hline $\mathrm{H}$ & $-1.3 \mathrm{E}-06$ & $5.64 \mathrm{E}-06$ & $-2.1 \mathrm{E}-06$ \\
\hline $\mathrm{H}$ & $-5 \mathrm{E}-06$ & $-3.8 \mathrm{E}-06$ & $-2.5 \mathrm{E}-06$ \\
\hline $\mathrm{H}$ & $1.66 \mathrm{E}-06$ & $-2.4 \mathrm{E}-06$ & $6.64 \mathrm{E}-06$ \\
\hline $\mathrm{H}$ & $2.65 \mathrm{E}-06$ & $3.94 \mathrm{E}-06$ & $-2.9 \mathrm{E}-06$ \\
\hline $\mathrm{H}$ & $-4.9 \mathrm{E}-06$ & $1.12 \mathrm{E}-06$ & $1.52 \mathrm{E}-07$ \\
\hline $\mathrm{H}$ & $4.78 \mathrm{E}-06$ & $-1.9 \mathrm{E}-06$ & $3.37 \mathrm{E}-06$ \\
\hline $\mathrm{H}$ & $3.52 \mathrm{E}-06$ & $-1.8 \mathrm{E}-06$ & $-5.4 \mathrm{E}-06$ \\
\hline $\mathrm{H}$ & $-1.8 \mathrm{E}-07$ & $-5.3 \mathrm{E}-07$ & $-6.1 \mathrm{E}-06$ \\
\hline $\mathrm{H}$ & $-9.7 \mathrm{E}-06$ & $-3.5 \mathrm{E}-06$ & $-5.7 \mathrm{E}-06$ \\
\hline
\end{tabular}

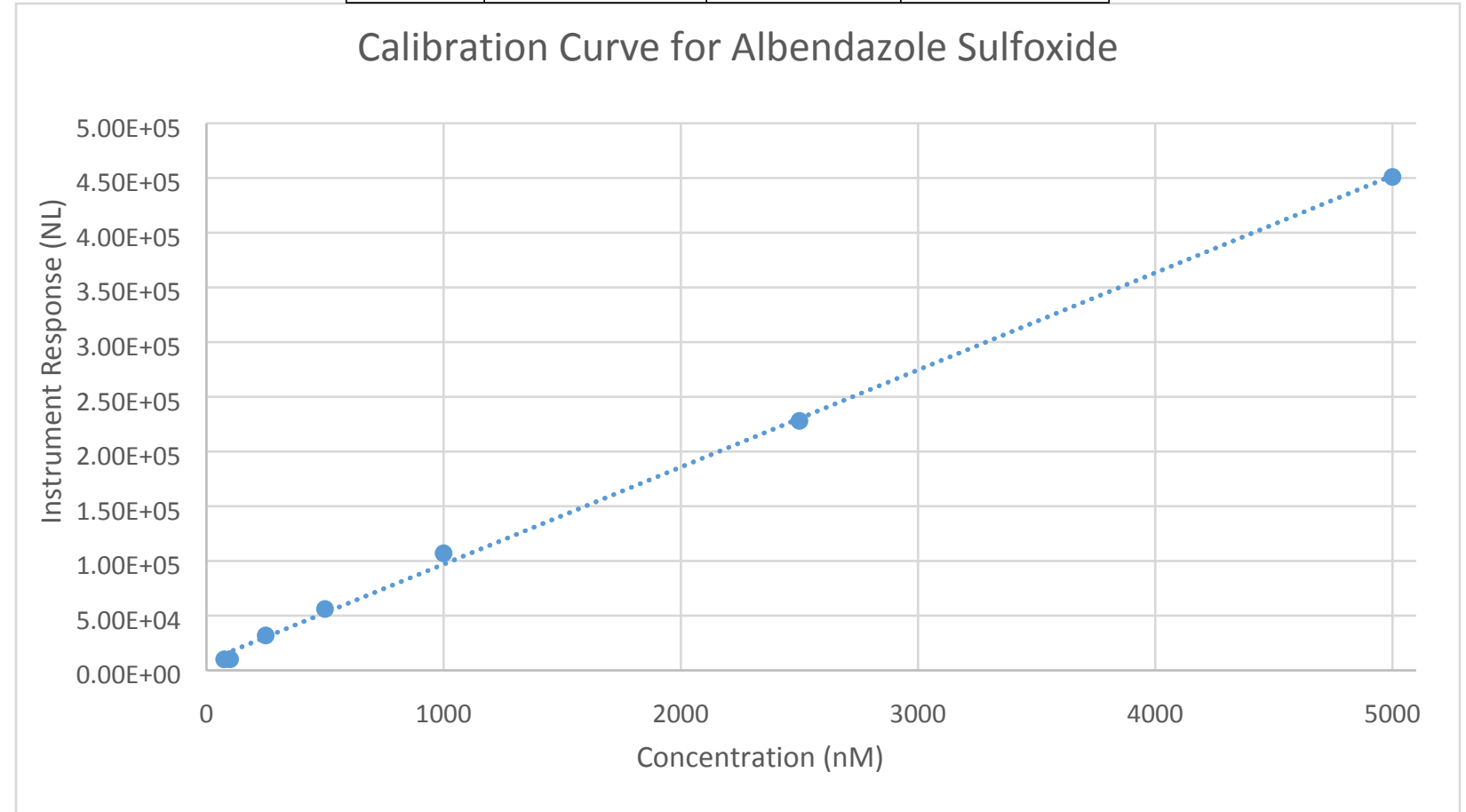

Figure S11. Calibration curve for albendazole sulfoxide showing the correlation between the mass spectrometer instrument response (in normalized level "NL") and the concentration of the analyte. The first two data points are for concentrations of 75 and $100 \mathrm{nM}$, respectively. The calibration curve is highly linear $\left(R^{2}=0.9988\right)$, showing a good correlation between the concentration of albendazole sulfoxide and the mass spectrometer response. The first two data points (75 and $100 \mathrm{nM}$ ) have nearly identical 
instrument responses, demonstrating that there is no longer a close correlation between concentration and instrument response below $100 \mathrm{nM}$, which was determined to be the instrument limit of detection.

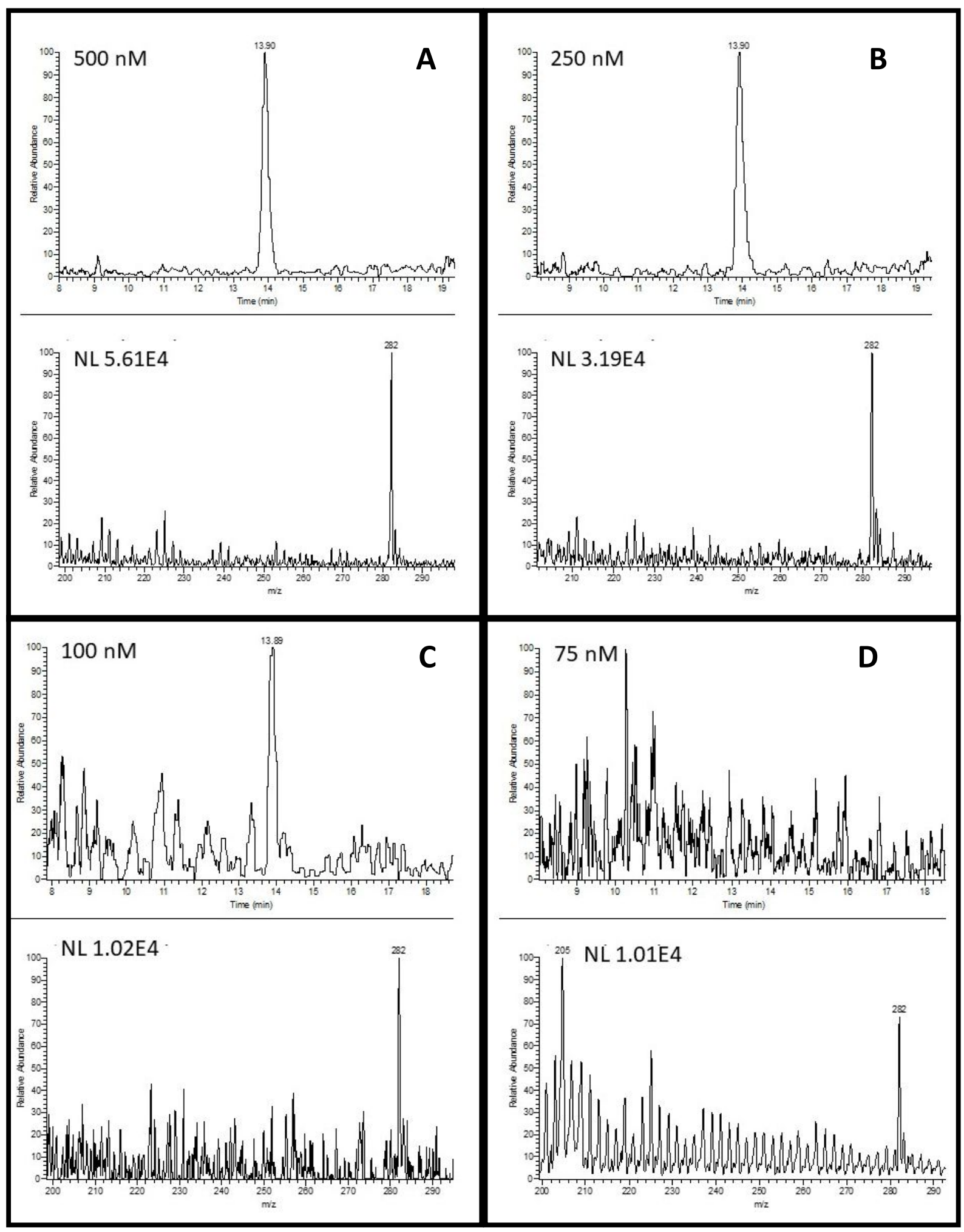


Figure S12. (A) - (D) Four sets of data showing an HPLC chromatogram (top of each data set) and a mass spectrum (bottom of each data set) for albendazole sulfoxide at four different concentations. The HPLC analyte peak eluted at $13.9 \mathrm{~min}$ and the analyte ion is seen at $\mathrm{m} / \mathrm{z} 282$ in the mass spectra. $100 \mathrm{nM}$ (in part C) is the lowest concentration at which both the HPLC chromatogram and the MS spectrum have a signal-to-noise ratio of at least 3. Each mass spectrum was an average of 125 - 150 scans. 


\begin{abstract}
Aberrant Result
One significant aberrant result was noted during the analysis of the analyte olanzapine. Olanzapine is a polyfunctional drug molecule containing several amino groups but no sulfoxide, $\mathrm{N}$-oxide, or sulfone functionalities. Therefore, protonated olanzapine was expected to show no reactivity towards TDMAB, as has been reported previously when using higher concentrations of the compound. ${ }^{4}$ However, it was discovered here that at sufficiently low concentrations $(\leq 100$ $\mathrm{nM}$ ), protonated olanzapine reacted with TDMAB to form a non-negligible adduct that had lost one DMA molecule. While the exact reason for this reaction is still unknown, the current hypothesis is that the reactivity arises from an unexpected protomer that is only preserved in the gas phase at low analyte concentrations.
\end{abstract}

